\title{
Better, Stronger, Faster: Why Add Fields to Syndromic Surveillance? New Jersey, 2015
}

\section{Pinar Erdogdu*, Teresa Hamby and Stella Tsai}

New Jersey Department of Health, Trenton, NJ, USA

\section{Objective}

Describe the inclusion of triage notes into a syndromic surveillance system to enhance population health surveillance activities.

\section{Introduction}

In New Jersey, real-time emergency department (ED) data are currently received from EDs by Health Monitoring Systems Inc.'s (HMS) EpiCenter, which collects, manages and analyzes ED registration data for syndromic surveillance, and provides alerts to state and local health departments for surveillance anomalies.

EpiCenter receives pre-diagnostic chief complaint data from 78 of 80 acute care and satellite EDs. The need for more specific information raises the possibility that other data elements from EDs such as triage notes can be of utility in detecting outbreaks without a significant delay [1]. This study evaluates the inclusion of triage notes in EpiCenter to detect a recent increased usage of synthetic cannabinoids. At the time of this evaluation, three New Jersey hospitals were providing triage notes in their EpiCenter data.

\section{Methods}

In April 2015, the New Jersey Poison Information and Education System (NJPIES) reported an increase in calls to their center for consultation regarding synthetic cannabinoid reactions in ED patients. This increase in calls resembled an outbreak [2], so the Department of Health (DOH) surveillance staff used the opportunity to compare chief complaints and triage notes to call data provided by NJPIES.

NJDOH created a custom classification in EpiCenter to detect synthetic cannabinoid-related ED visits using chief complaint data. DOH staff included the keywords "black magic", "black mamba", "cloud 9", "cloud 10", "incense", "k2", "legal high", "pot potpourri”, "spice", "synthetic marijuana", "voodoo doll", "wicked x", and "zombie" which were obtained from the New York City Department of Health and Mental Hygiene. Staff also included the keywords, "agitation", "k-2", "moon rocks", "seizure", "skunk", and "yucatan" to characterize the related event.

$\mathrm{NJDOH}$ performed a text search comparison using the same keywords in the triage notes field from three EDs currently providing that data to evaluate the synthetic cannabinoid related ED visits.

\section{Results}

Using the keywords, out of 50 NJPIES calls, 18 (36\%) were identified via chief complaint data and $32(64 \%)$ of the ED visits were not captured due to the non-specificity of the keywords used. Among the 18 visits, the most common keywords were "seizure" and "marijuana".

Of the 50 calls, 6 ED visits were admitted to hospitals that submitted triage notes data in EpiCenter. Using the same keywords in a triage notes query, 5 (83\%) out of 6 ED visits were identified. The most common keywords were "k2" and "marijuana".

Overall, based on the chief complaints and triage notes from these three EDs (figure 1 and table 1), triage notes are able to provide more information about ED visits related to synthetic cannabinoid between March 27 and May 16, 2015.

\section{Conclusions}

Review of NJPIES synthetic cannabinoid calls suggests that triage notes in syndromic surveillance systems included more specific keywords than the chief complaints and captured most of the calls related to synthetic cannabinoid. Triage notes inclusion has been initiated in New Jersey. This new data source will provide vital information to syndromic surveillance, which is expected to lead to earlier detection and response to health events like Ebola, Enterovirus D68, and drug overdose surveillance.

Table 1: Number of ED visits from 3 hospitals submitted both triage notes and chief complaint data between March 27, 2015 and May 16, 2015. Triage notes captured more ED visits compared to chief complaints.

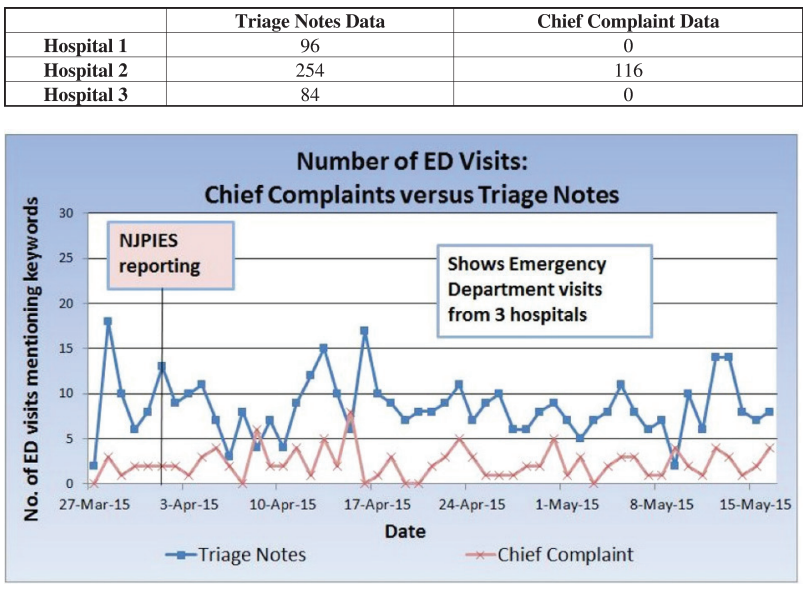

Figure 1: Number of ED visits by data type from three hospitals submitting both triage notes and chief complaint data between March 27, 2016 and May 16,2015 . Related to synthetic cannabinoid use, triage notes included more visits most days then cheif complaints. On some dates, cheif complaints included more ED visits.

\section{Keywords}

Syndromic Surveillance; EpiCenter; New Jersey

\section{Acknowledgments}

Bruce E. Ruck, PharmD, RPh (NJPIES), Elizabeth Kostial (HMS), New York City Department of Health and Mental Hygiene.

\section{References}

1. Walsh A, Fowler B. Using Triage Notes to Refine Syndromic Surveillance: An Ebola Case Study. Paper presented at: 2015 CSTE Annual Conference; 2015 May 14-18; Boston, MA.

2. Schwarz A. Potent 'Spice' Drug Fuels Rise in Visits to Emergency Room. The New York Times.2015 Apr 24.Available from http:/ www.nytimes.com/2015/04/25/health/surge-in-hospital-visits-linkedto-a-drug-called-spice-alarms-health-officials.html

\section{*Pinar Erdogdu}

E-mail: pinar.erdogdu@doh.state.nj.us 